\title{
Prediction of the particle production in pp collisions with the MPD detector at NICA collider
}

\author{
$K$. Shtejer ${ }^{1,2, *}$ for the MPD Collaboration \\ ${ }^{1}$ Joint Institute for Nuclear Research (JINR), Dubna, Russia \\ ${ }^{2}$ Centro de Aplicaciones Tecnológicas y Desarrollo Nuclear (CEADEN), Havana, Cuba
}

\begin{abstract}
The proton-proton collisions have been extensively used as a baseline reference for nucleus-nucleus collisions. In this work, Monte Carlo simulations of inelastic head-on pp interactions at $\sqrt{s}=6-25 \mathrm{GeV}$ were performed to study the possibilities of the Multi-Purpose Detector (MPD) to register data from small systems at the energy range of NICA. For that purpose the Monte Carlo models EPOS 1.99 and UrQMD 3.4 were used as event generators to analyse the charge particle yield from pp collisions in the framework of MpdRoot. The production of protons, pions and kaons from pp collisions was studied through their mean multiplicity, rapidity spectra and particle ratios as function of the collision energy. The yield of $\Lambda$ hyperons was also estimated by means of pure Monte Carlo and the reconstruction of the simulated data in MPD. The comparison with recent data from NA61/SHINE experiment is presented.
\end{abstract}

\section{Introduction}

The NICA accelerator will provide colliding beams with masses ranging from protons to gold $\left(\mathrm{Au}^{79+}\right)$. The collision energy range for $\mathrm{Au}^{79+}$ is intended to be $\sqrt{s_{\mathrm{NN}}}=4-11 \mathrm{GeV}$ per nucleon pair, while for protons is up to $27 \mathrm{GeV}$. The MPD is the main collision detector of NICA and has the barrel geometry typical of collider detectors [1]. The installation and tests of the main barrel part of MPD, composed by the TPC (Time Projection Chamber), TOF (Time of Flight system) and ECal (Electromagnetic Calorimeter) is scheduled to finish in 2020, together with the FHCal (Forward Hadron Calorimeter) and the FFD (Fast Forward Detector). The proton-proton collisions constitute an essential prerequisite in the MPD/NICA research program, either as a detector performance tool, as a baseline for nucleus-nucleus collision analyses and for a better understanding of light collision systems. We present a prediction of the charged particle yield in pp collisions using simulated data based on two models, and also the neutral $\Lambda$ hyperon yield obtained by Monte Carlo (MC) simulation and the reconstruction in the central barrel part of MPD.

\section{Simulations in MPD}

The framework used to perform simulations of inelastic pp interactions in MPD is the dedicated software MpdRoot where the Monte Carlo step makes use of GEANT tools to transport the particles through the detector material as realistic as possible. The reconstruction begins

\footnotetext{
*e-mail: kshtejer@jinr.ru
} 
with cluster/hit finding, thus giving input to the Kalman Filter technique to reconstruct the tracks that are accordingly used for the vertex finding. The reconstructed tracks are associated with their corresponding MC particle [6]. In the present stage of MPD all tracks are propagated through the TPC and, when possible, they are matched with the hits reconstructed in the TOF detector. This information is later used for the particle identification (PID) procedure.

From previous simulations of pp collision in MPD [7], we know that the track reconstruction efficiency in the TPC acceptance approaches $100 \%$ for $p_{\mathrm{T}}>0.2 \mathrm{GeV} / c$. It goes down to about $75 \%$ at $p_{\mathrm{T}}=0.1 \mathrm{GeV} / c$ and $50 \%$ at $p_{\mathrm{T}}=0.05 \mathrm{GeV} / c$. In a large pseudorapidity $(\eta)$ range the efficiency approaches to $100 \%$ for primary particles and $90 \%$ for secondaries, becoming degraded at extreme $\eta$ values. The $p_{\mathrm{T}}$ resolution, considering all primary tracks reconstructed in the TPC is typically better that $2.6 \%$ in the interval $p_{\mathrm{T}}=0.1-1.5 \mathrm{GeV} / c$. For $p_{\mathrm{T}}>1.5 \mathrm{GeV} / c$ there is few statistics.

With the aim of studying, by Monte Carlo, the charged particle multiplicity produced by pp collisions in MPD, events with single head-on inelastic pp interactions were generated with two models, EPOS 1.99 [2] and UrQMD 3.4 [3], and then propagated through MPD. EPOS 1.99 was selected by the fact of having showed a good description of recently reported NA61/SHINE experimental results of pp collisions at the CERN Super Proton Synchrotron (SPS) [4]. The collision energies reached at SPS constitutes a subrange of the expected pp $\sqrt{s}$ range in NICA.

\subsection{Mean multiplicity of charge particles}

The rapidity density distributions of $\pi^{ \pm}, K^{ \pm}, p$ and $\bar{p}$ produced in pp collisions were determined from MC simulations using EPOS 1.99 and UrQMD 3.4 at $\sqrt{s}=6-25 \mathrm{GeV}$. The model predictions were compared with the experimental results of NA61(SPS) for the energies where measurements were performed. The rapidity spectra of each particle specie, obtained at $\sqrt{s}=17.3 \mathrm{GeV}$ and normalised by the number of events, is shown in Fig. 1.

The rapidity spectra of charged particles in Fig. 1 reveal differences in the predictions of the models selected for this analysis, and regarding the published SPS measurements. The simulation with EPOS 1.99 agrees better with the experimental data. The UrQMD 3.4, instead, tends to underestimate $\pi^{ \pm}$in the whole rapidity range and $K^{+}$at mid-rapidity, and overestimates the yield of $p$ and $\bar{p}$. At forward rapidity, where the maximum proton yield is expected, both models are in disagreement with each other and with the SPS data. A similar behaviour was obtained for the other simulated collision energies, with enlarged discrepancies of UrQMD for $\pi^{ \pm}, K^{ \pm}$and $p$ as $\sqrt{s}$ decreases, and for $\bar{p}$ as $\sqrt{s}$ increases. The transverse momentum $\left(p_{\mathrm{T}}\right)$ spectra were also analysed in slices of rapidity (not shown here). EPOS 1.99 describes better the $p_{\mathrm{T}}$ experimental spectra. The main discrepancies from UrQMD 3.4 arise at $p_{\mathrm{T}}<0.5 \mathrm{GeV} / c$ for $\pi^{ \pm}$and $K^{ \pm}$, at $p_{\mathrm{T}}>0.5 \mathrm{GeV} / c$ for $\bar{p}$ and for the whole proton $p_{\mathrm{T}}$ spectrum.

The mean multiplicities of $\pi^{ \pm}, K^{ \pm}, p$ and $\bar{p}$ produced in inelastic pp collisions, predicted by MC simulations based on EPOS 1.99 and UrQMD 3.4 to generate inelastic pp events in MPD, were calculated by integrating the rapidity spectra all over $\sqrt{s}=6-25 \mathrm{GeV}$ (Fig. 2).

The consistency of EPOS 1.99 contrasts with the underestimation of $\pi^{ \pm}$and $K^{ \pm}$by UrQMD 3.4 along with its systematic overprediction of $p$ and $\bar{p}$ multiplicities. According to this simulation the mean proton multiplicity remains almost constant in the NICA energy range. The corresponding relative difference between each model and the SPS data is shown in the bottom plots. The difference between UrQMD 3.4 and the SPS data can reach up to $50 \%$ of the corresponding experimental data. The relative difference between the data and EPOS 1.99 mean multiplicities in general do not exceed $10 \%$, except for $K^{-}, p$ and $\bar{p}$ at $\sqrt{s}<8.8 \mathrm{GeV}$ where $28 \%$ difference was obtained. The statistical uncertainties of the mean 

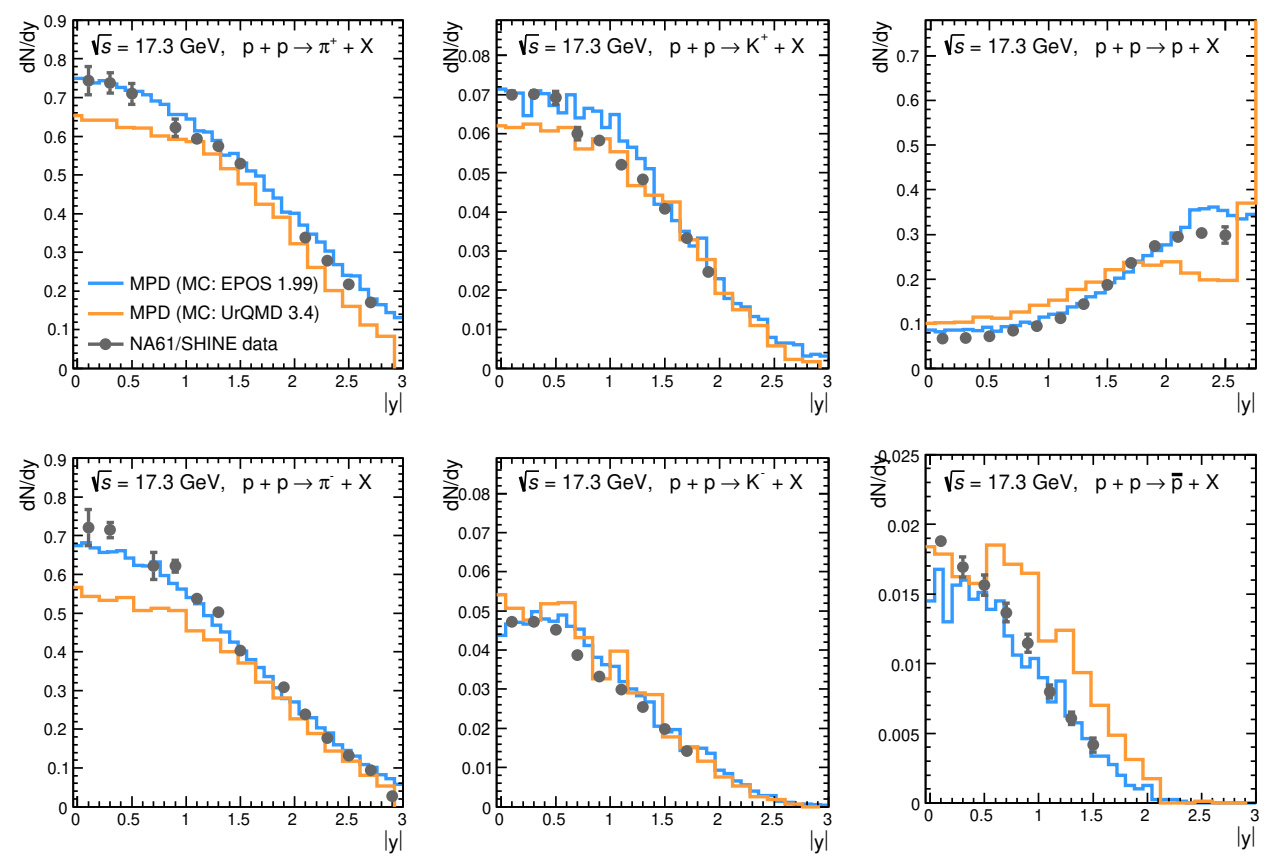

Figure 1. Monte Carlo simulations of the rapidity density distribution, normalised by the number of inelastic pp collisions at $\sqrt{s}=17.3 \mathrm{GeV}$, predicted by EPOS 1.99 [2] and UrQMD 3.4 [3] in MPD, and compared with NA61/SHINE measurements [4]

multiplicity values from the EPOS 1.99 simulations are lower that $0.5 \%$ while for UrQMD 3.4 are between $0.2 \%$ and $0.7 \%$. The simulations with EPOS were based on $10^{6}$ events while with UrQMD, which is quite consuming time, $10^{4}$ events were generated.

\subsection{Excitation function of kaon-to-pion ratio}

The kaon-to-pion ratio is a diagnostic tool to study the strongly interacting matter through the relative strangeness yield. In particular, the peak resembling to a horn structure in the $K^{+} / \pi^{+}$distribution observed in heavy ion collisions at $\sqrt{s} \approx 8 \mathrm{GeV}$, might be an indication of the onset deconfinement when is compared with lighter collision systems [5]. However, since most of tradicional models are unable to describe this effect, it requires a more detailed study, and NICA should be a good opportunity for that.

The excitation functions of the $K^{+} / \pi^{+}$and $K^{-} / \pi^{-}$ratios were simulated for inelastic pp collisions in MPD at mid-rapidity. The predictions of EPOS 1.99 and UrQMD 3.4 are compared, in Fig. 3, with experimental results from NA61/SHINE. The model EPOS 1.99 is more consistent with the NA61/SHINE data over the whole analysed energy range and it describes a near-constant $K^{+} / \pi^{+}$ratio from $\sqrt{s}=8 \mathrm{GeV}$ on. The measurements from NA61/SHINE constitute a convenient referral point since they provide accurate experimental results in the NICA energy range. The UrQMD 3.4 model underpredicts $K^{+} / \pi^{+}$ratio at collision energies under $12 \mathrm{GeV}$, and overpredicts the $K^{-} / \pi^{-}$ratio over the NICA energy range, even approaching to the heavy ion collision measurements. The horn structure is not present in $K^{-} / \pi^{-}$ excitation function (Fig. 3, right). 

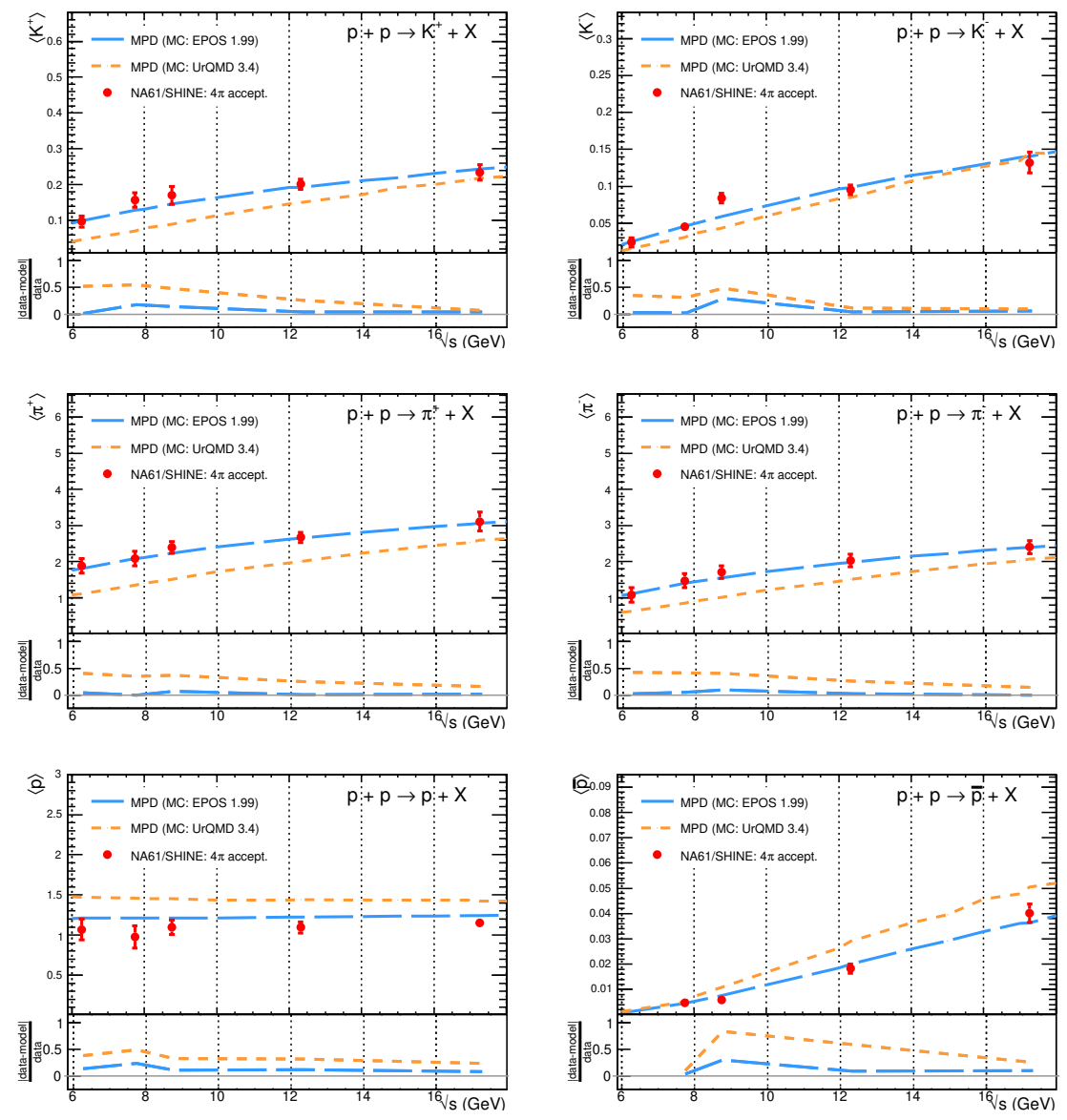

Figure 2. The mean multiplicity of $\pi^{ \pm}, K^{ \pm}, p$ and $\bar{p}$ produced in inelastic pp collisions from Monte Carlo simulations in MPD. The predictions of EPOS 1.99 [2] and UrQMD 3.4 [3] are compared with NA61/SHINE measurements [4]. The relative differences with the SPS data are in the bottom plots

\section{Yield of $\Lambda$ hyperon}

The strange particle analysis in NICA, where the maximal baryon density takes place, is one of the main goals of the MPD physics program. In heavy ion collisions there is an enhancement of the hyperon yield per nucleon, relative to pp collisions, that has been predicted as a signal of the onset of deconfinement. This enhancement increases with the strange content of the hyperon. The contribution of the strange baryons to the global strange content dominates at low collision energies and loses importance at high energies. The hyperon production in pp interactions has been widely addressed by several experiments over the years, however they are characterised by a lack of statistics and large differences between them.

The yield of $\Lambda$ hyperon in pp collisions in MPD, was here determined by MC simulations at different energies in the range $\sqrt{s}=6-25 \mathrm{GeV}$ using the EPOS 1.99 generator. In Fig. 4 our simulation results are compared with existing experimental data, including the measurement from NA61/SHINE which has so far the smallest uncertainty [8]. The predictions of 

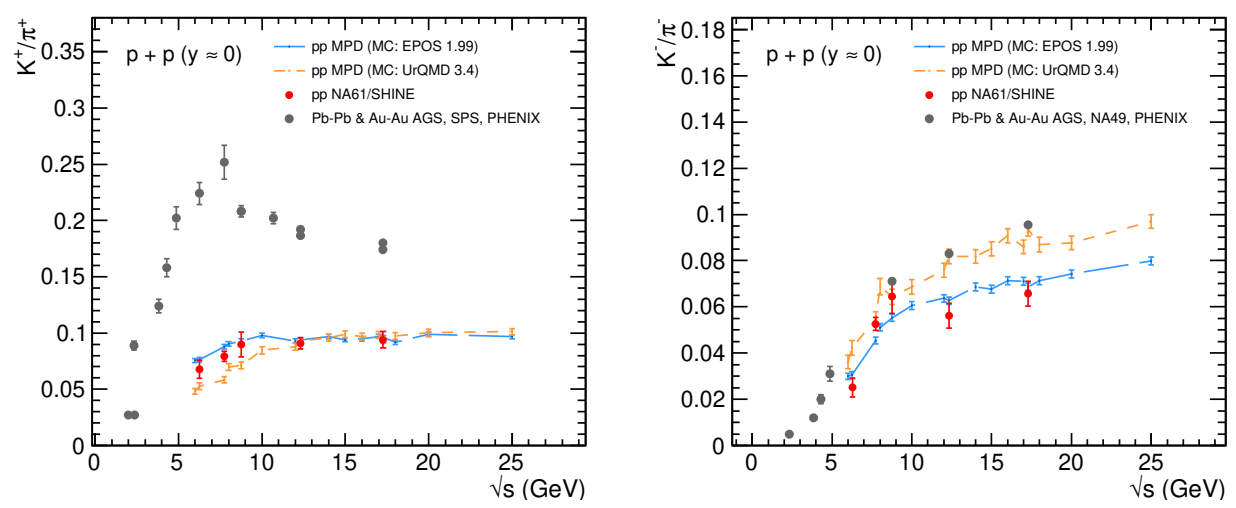

Figure 3. The excitation function of $K^{+} / \pi^{+}$and $K^{-} / \pi^{-}$, predicted by EPOS 1.99 and UrQMD 3.4 in MPD. The simulation results are compared with NA61/SHINE measurements. A compilation of experimental data from $\mathrm{Pb}-\mathrm{Pb}$ and $\mathrm{Au}-\mathrm{Au}$ is represented by grey circles to show the "horn effect" as reference. The blue and orange lines through the simulated data are to guide the eye, and the vertical bars indicate statistical uncertainties of the simulated ratios

the simulations in MPD, based on the EPOS 1.99, show a smooth increase with $\sqrt{s}$ and are in acceptable agreement with the bulk of data.

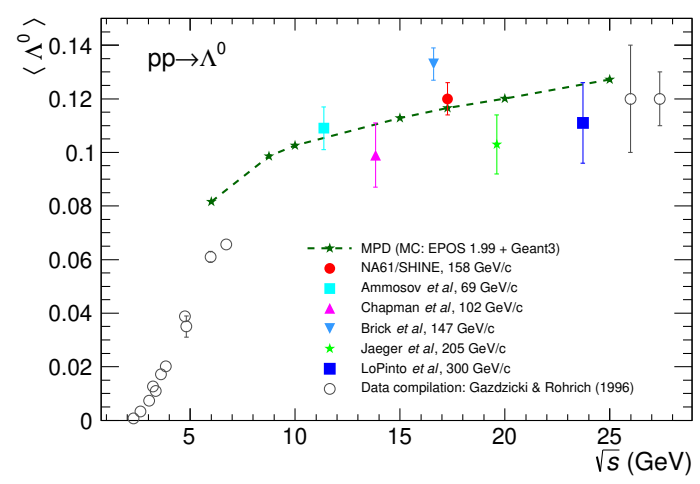

Figure 4. The mean multiplicity of $\Lambda$ hyperon vs. $\sqrt{s}$, produced in inelastic pp collisions from Monte Carlo simulations in MPD

The reconstruction and extraction of the $\Lambda$ signal was carried out for each simulated collision energy. Candidates of pairs of positive and negative tracks identified as $p$ and $\pi^{-}$ and decayed from a primary particle were identified by applying secondary vertex finding technique with standard topological cuts. The $\Lambda$ signal was extracted from the combinatorial background found in the invariant mass distribution, through a fitting procedure based polynomial and Gaussian functions for the background and peak respectively. The invariant mass distribution of primary $\Lambda$ hyperons from pp interactions at $\sqrt{s}=17.3 \mathrm{GeV}$ is shown in the first plot of Fig. 5. The other five plots of Fig. 5 show the reconstructed $\Lambda$ hyperon in bins of $p_{\mathrm{T}}$. 

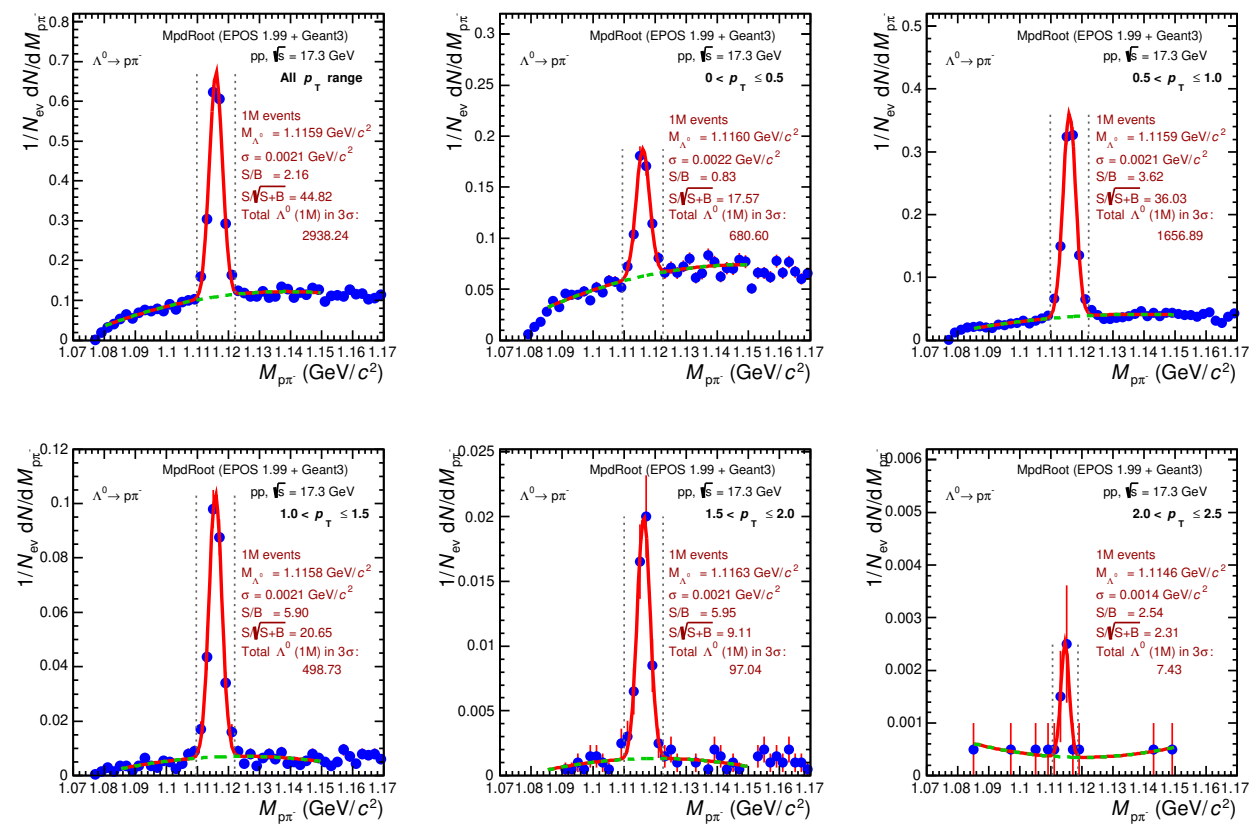

Figure 5. The $\Lambda$ hyperon reconstruction in bins of $p_{\mathrm{T}}$, produced in inelastic pp collisions at $\sqrt{s}=17.3$ $\mathrm{GeV}$. The first plot corresponds to the $\Lambda$ reconstructed over the whole $p_{\mathrm{T}}$ range

This reconstruction of $\Lambda$ hyperon is constrained by the geometrical acceptance limits imposed the PID procedure, so that the reconstructed $\Lambda$ yield corresponds to the pseudorapidity limits of TOF $(|\eta|<1.3)$.

From Fig. 5, it can be seen that despite the low statistics, typical of pp collisions, the $\Lambda$ signal can be reconstructed in MPD. The maximum value of $\Lambda$ yield, the highest significance $(S / \sqrt{S+B})$ and the better $S / B$ value are obtained for $p_{\mathrm{T}}$ between 0.5 and $1 \mathrm{GeV}$.

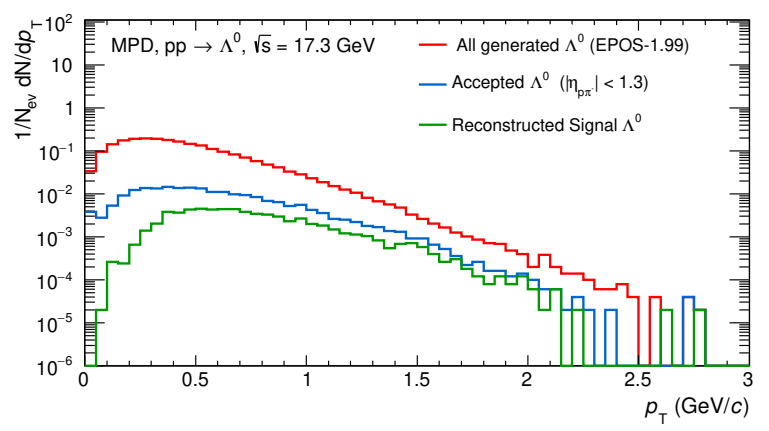

Figure 6. The $p_{\mathrm{T}}$ spectrum of $\Lambda$ hyperons. See text for details

In Fig. 6 is shown the $p_{\mathrm{T}}$ spectrum of all $\Lambda$ hyperons generated by EPOS 1.99 and propagated with GEANT through the detector MPD (red), the accepted $\Lambda$ hyperons during the MC 
simulations by the decays of $p \pi^{-}$pairs within $|\eta|<1.3$ (blue) and the finally reconstructed signal of $\Lambda$ hyperons from the $p \pi^{-}$invariant mass distribution (green).

\section{Conclusions}

The yield of $\pi^{ \pm}, K^{ \pm}, p$ and $\bar{p}$ produced in inelastic pp interactions at $\sqrt{s}=6-25 \mathrm{GeV}$, was predicted by MC simulations based on two models, EPOS 1.99 and UrQMD 3.4, used as event generators and the GEANT tools to propagate particles through the MPD structure. Experimental data on charged particle multiplicity in the NICA energy range, which meanwhile became available from NA61 (SPS), were used as reference for comparison. According to our simulations, EPOS 1.99 provides a reasonable description of the available measurements from SPS, while UrQMD 3.4 shows disagreements. From the comparison with pp SPS data the kaon-to-pion ratio is well described by EPOS 1.99. However UrQMD underpredicts $K^{+} / \pi^{+}$data at $\sqrt{s}<12 \mathrm{GeV}$, thus it is not suitable for studies of pp collisions at the collision energies where the phase transition is expected to occur. Monte Carlo simulations of $\Lambda$ hyperon produced in pp collisions in MPD using the EPOS 1.99 generator at $\sqrt{s}=6-25 \mathrm{GeV}$, describe quite well the experimental data reported in the literature for the same energies. The reconstruction of this particle in the MPD geometrical acceptance given by TOF and TPC detectors, and using the particle identification method implemented in MpdRoot, bring forth a well defined signal. The study of this signal reconstruction in different $p_{\mathrm{T}}$ bins reveals good possibilities to study the hyperon production from pp collisions in MPD.

\section{References}

[1] V. Golovatyuk et al., Eur. Phys. J. A 52, 212 (2016)

[2] K. Werner, F. Liu and T. Pierog, Phys. Rev. C 74, 044902 (2006)

[3] S. Bass et al., Prog. Part. Nucl. Phys. 41, 255 (1998)

[4] The NA61/SHINE Collaboration, A. Aduszkiewicz et al., Eur. Phys. J. C 77, 671 (2017)

[5] M. Gazdzicki and M.I. Gorenstein, Acta. Phys. Polon. B 30, 2705 (1999)

[6] K. Gertsenberger et al., Eur. Phys. J. A 52, 214 (2016)

[7] K. Shtejer et al., Acta Phys. Pol. B Proc. Suppl. 11(4), (2018)

[8] The NA61/SHINE Collaboration, A. Aduszkiewicz et al., Eur. Phys. J. C 76, 198 (2016) 